\title{
Clima laboral y Evaluación del Desempeño a Jefes de área en Hospitales del MINSA, Región Arequipa, 2017.
}

Work climate and Performance Evaluation to Area Chiefs in MINSA Hospitals, Arequipa Region, 2017.

\author{
Giselle Milagros Alemán Espinoza
}

Universidad Nacional de San Agustín. Arequipa Perú.

INFORMACIÓN RESUMEN

\author{
Historia del Artículo \\ Recepción: 02/06/2019 \\ Revisión: 15/07/2019 \\ Aceptación: 20/07/2019

\section{Palabras Clave} \\ Clima Laboral, Evaluación del \\ Desempeño, Región Arequipa. \\ UNSA, CIENCIACTIVA, \\ CONCYTEC.
}

\section{Key Words \\ Work Climate, Performance Evaluation, Region Arequipa, UNSA, CIENCIACTIVA, CONCYTEC.}

\section{DOI}

https://doi.org/10.35286/veritas. v20i1.224
El objetivo de la investigación es identificar la relación entre el clima organizacional y la evaluación del desempeño. Se efectuó una evaluación del desempeño de 360 grados a 55 Jefes de Área de Hospitales en la Región Arequipa, 38 varones y 17 mujeres, con personal a cargo, con un total de 385 evaluaciones, aplicadas a sus superiores, pares y subordinados con un Cuestionario de Evaluación del Desempeño (elaboración propia) y se utilizó la Escala de Clima Laboral de Sonia Palma Carrillo (CL-SPC) para medir el clima laboral. Los resultados obtenidos por el análisis estadístico muestran que no existe una relación significativa entre el clima laboral y el desempeño laboral, sin embargo, si existen relaciones significativas entre las variables y sus indicadores, por separado. Se realiza un análisis de los resultados, discusión, conclusiones y se dan recomendaciones para el sector salud.

\begin{abstract}
The objective of the research is to identify the relationship between the organizational climate and performance evaluation. An evaluation of the performance of 360 degrees was made to 55 Heads of Area of Hospitals in the Arequipa Region, 38 men and 17 women, with personnel in charge, with a total of 385 evaluations, applied to their superiors, peers and subordinates with a Questionnaire of Performance Evaluation (own elaboration) and the Sonia Palma Carrillo Work Climate Scale (CL-SPC) was used to measure the work climate. The results obtained by the statistical analysis show that there is no significant relationship between the work environment and work performance, however, if there are significant relationships between the variables and their indicators, separately. An analysis of the results, discussion, conclusions and recommendations are given for the health sector.
\end{abstract}

\section{INTRODUCCIÓN}

Nuestro contexto laboral evoluciona constantemente. Competir ya no nos hará mejores localmente, sino que compartir, es lo que nos convertirá en útiles e importantes para la humanidad, en ciudadanos del mundo, por lo que se ha vuelto indispensable, pensar en el clima laboral y en la evaluación del desempeño laboral. Según Puchol (2007), la evaluación del desempeño es "un procedimiento continuo, sistemático, orgánico y en cascada, de expresión de juicios acerca del personal de una empresa en relación con su trabajo habitual, y que pretende sustituir a los juicios ocasionales y multicriterio". Por lo que en esta investigación se indaga acerca del desempeño laboral con un enfoque de 360 grados a fin de conocer con mayor amplitud a los trabajadores. Smith, Gunzenhauser y Fielding (2010) destacan, que si la evaluación del desempeño, está claramente relacionada con las normas y las competencias, puede ser una ayuda valiosa para retener a los empleados, mediante la promoción de un ambiente de trabajo, donde se reconocen los logros y se celebran los hitos en el crecimiento profesional de un empleado. En el caso de la relevancia de la investigación

\section{Correspondencia:}

Giselle Milagros Alemán Espinoza

giselle.aleman1@gmail.com sobre el clima laboral, tenemos aportes frondosos por varias disciplinas científicas, todas ellas se centran en la mejora de la calidad de vida del trabajador. Tanto es así, que el clima laboral es determinante en la forma que toma una organización, en sus relaciones y decisiones internas y externas, por lo que Litwin y Stinger (1968) postulan la existencia de dimensiones que explicarían el clima existente en una determinada empresa (citado por Robbins, 1999). Por esta razón muchas organizaciones reconocen que uno de sus activos fundamentales y tal vez uno de los que más rápido pueda perderse es la gestión del talento humano. Para estar seguros de la solidez de su recurso humano, las instituciones necesitan medir y mejorar constantemente su clima organizacional que está entrelazado con la motivación individual y de los grupos laborales y en el tiempo tener repercusiones positivas o negativas frente al comportamiento organizacional, la satisfacción y el desempeño laboral. El proceso de tareas que hay que cumplir para consolidar todas estas temáticas no es simple, sino complejo, y añade valor estratégico a las empresas contemporáneas, en algunos casos esto es medido por empresas, como Great Place to Work ${ }^{\circledR}$ por medio de sus analistas que evalúan las organizaciones y seleccionan los mejores lugares de trabajo para su posible inclusión en sus listas sobre la base de dos de sus herramientas de evaluación, con ello se comprende la experiencia en el lugar de trabajo y la efectividad de las 
prácticas que impactan en la cultura del lugar de trabajo, lo cual es mérito para las personas y las organizaciones, garantizando premios y seguramente grandes perspectivas de desarrollo y negocios. El clima laboral u organizacional tiene una significativa relación en la determinación de la cultura organizacional, donde se configura el patrón general de conductas, creencias y valores sobrellevados por los miembros de una institución. El objetivo del presente estudio fue hallar la correlación existente entre el clima laboral y el desempeño laboral en los trabajadores del sector salud con rango de jefaturas o encargaturas en los hospitales del MINSA de la Región Arequipa y entre los indicadores del clima laboral con el desempeño laboral general. En este sentido, los indicadores del clima laboral analizados fueron cinco: autorrealización o realización personal, involucramiento laboral, supervisión, comunicación y condiciones laborales; y en el caso del desempeño laboral fueron doce: planificación, responsabilidad, iniciativa, oportunidad, calidad del trabajo, confiabilidad y discreción, relaciones interpersonales, cumplimiento de las normas, liderazgo, orientación a los resultados, adaptación al cambio y toma de decisiones.

\section{MATERIAL Y MÉTODOS}

El enfoque de la investigación es cuantitativa, de tipo no experimental, correlacional, transversal. El universo estuvo representado por todos los trabajadores del sector salud de la Región Arequipa pertenecientes al Ministerio de Salud del Perú (MINSA). La población estuvo conformada por todos los jefes de área de los Hospitales de la Región Arequipa, pertenecientes al MINSA, la muestra fue de 55 Jefes en total, respecto al sexo de los cuales 38 fueron personas de sexo masculino y 17 personas de sexo femenino; en relación a la edad, se contó con 3 rangos edades clasificados para la parte estadística, y también se consideró la modalidad del vínculo laboral, siendo contratados o nombrados. En relación a los hospitales también se delimitaron los sujetos participantes, para las evaluaciones de la siguiente manera: por cada jefe de área (01) una autoevaluación, luego su superior lo evaluó, (01) una evaluación del inmediato superior siendo el Director del Hospital o el Sub Director de Hospital, así como (02) dos evaluaciones de sus pares o compañeros colegas jefes de áreas, y (03) tres evaluaciones de sus subordinados o sub alternos quienes trabajan bajo su mando, en total fueron 385 evaluaciones.

La recolección de datos, se realizó con 2 instrumentos: la Escala de Clima Laboral de Sonia Palma Carrillo (ECLSPC) y la Escala de Clima Laboral CL - SPC de Sonia Palma (1999) y el Cuestionario de Desempeño Laboral (2017), elaborado en la presente investigación. Además, se ha utilizado el coeficiente Alfa de Cronbach para la confiabilidad estadística y el coeficiente de Pearson para medir la relación y correlación entre las variables propiamente dichas. Para fines de validación de los instrumentos se realizó una prueba piloto en el Hospital Militar III DE Arequipa, obteniéndose niveles óptimos, en el caso del Cuestionario de Desempeño Laboral, con anterioridad se elaboró, formuló y aprobó, mediante juicio de expertos, siendo en total 10 profesionales especializados, donde también colaboró el Gerente Regional de Salud del Gobierno Regional de Arequipa, entre otros.

\section{RESULTADOS}

Los resultados de validez dados mediante el coeficiente de Alfa de Cronbach son óptimos, se utilizó el coeficiente de correlación de Pearson, notándose que cada instrumento utilizado por separado presenta relaciones significativas entre sus indicadores y sus valores en general.

Tabla 1: Hospital - Clima Laboral General - Desempeño Laboral General en Porcentajes

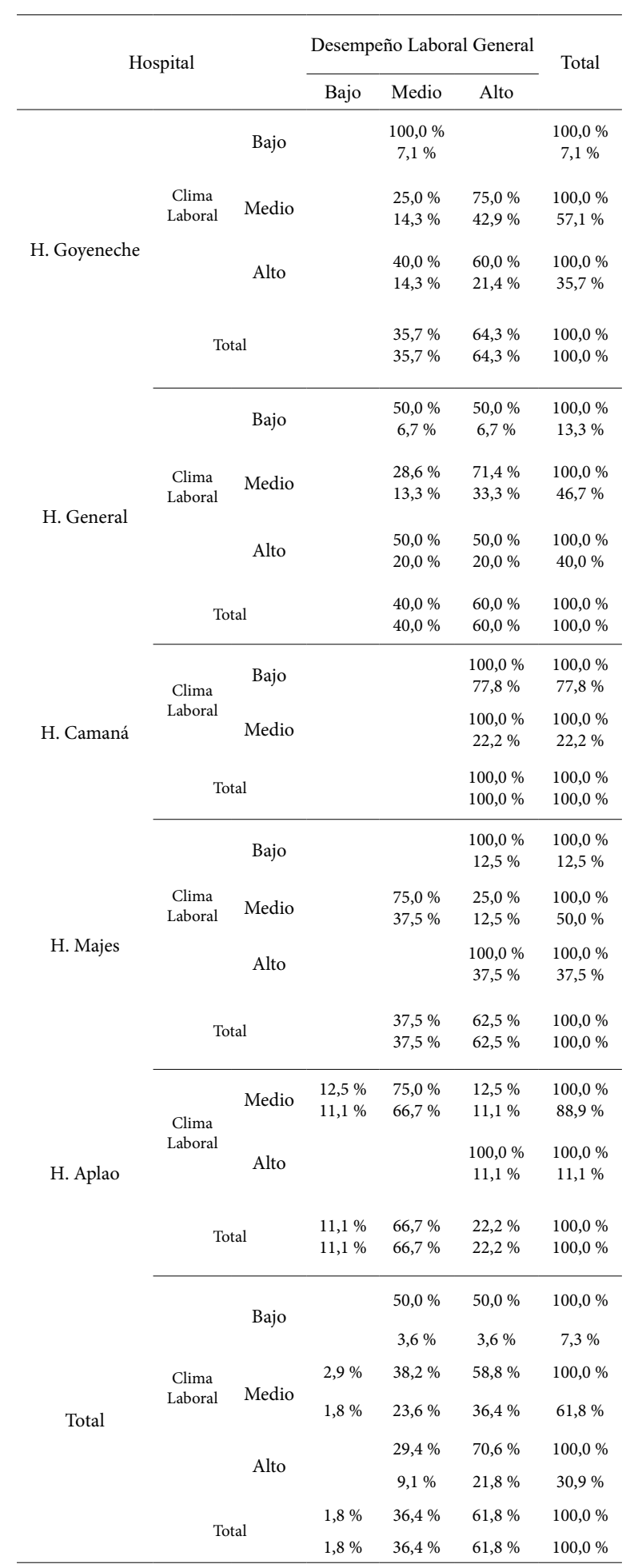


En la tabla 1, se analiza en porcentajes la relación entre los hospitales, y las variables clima laboral y el desempeño laboral, de lo cual se evidencia que entre las variables en forma general existen tres niveles. En el caso de los porcentajes en primer término se refieren al porcentaje dentro del clima laboral y en segundo término al porcentaje del total, por lo que por cada nivel del clima laboral hay dos porcentajes, lo cual también se toma en cuenta para el análisis de las siguientes tablas.

En relación al Hospital Goyeneche, se aprecia que existen más niveles medios y altos, en cuanto al clima laboral general y al desempeño laboral general, resaltando que no existe un nivel bajo de desempeño laboral general. En cuanto al Hospital General, se estima que existe mayor predominancia de niveles altos y luego niveles medios, en cuanto al clima laboral general y al desempeño laboral general, además, nuevamente no existe un nivel bajo de desempeño laboral general. Respecto al Hospital de Camaná, se valora que no existen niveles bajos de clima laboral general, y en cuanto al desempeño laboral general no existen niveles bajos ni medios, considerando que existen más niveles altos en ambos aspectos. En el caso del Hospital de Majes, se considera que no existen niveles bajos de desempeño laboral general, y si niveles medios y altos, y en cuanto al clima laboral general este es mayoritariamente de nivel medio. Finalmente, en el Hospital de Aplao, se ha encontrado no existen niveles bajos de clima laboral general y que el desempeño laboral general es en gran medida de nivel medio.

En general, se ha determinado que existen más niveles altos, seguido de niveles medios, y en menos del $2 \%$ del total a niveles bajos, en cuanto al clima laboral general y también respecto al desempeño laboral general en todos los Hospitales de la Región Arequipa pertenecientes al MINSA.

Tabla 2: Correlación entre los indicadores del Clima Laboral y el Desempeño Laboral General

\begin{tabular}{|c|c|c|c|c|c|c|c|c|}
\hline & & Clima & C. Realización & C. Involucramiento & C. Supervisión & C. Comunicación & C. Condiciones & Desempeño \\
\hline & $\begin{array}{l}\text { Correlación de } \\
\text { Pearson }\end{array}$ & 1 &, $911^{* *}$ &, $905^{\star *}$ &, $926^{\star *}$ &, $902^{\star *}$ &, $914^{* *}$ &, 010 \\
\hline \multirow[t]{3}{*}{ Clima } & Sig. (bilateral) & &, 000 &, 000 &, 000 &, 000 &, 000 & 944 \\
\hline & $\mathrm{N}$ & 55 & 55 & 55 & 55 & 55 & 55 & 52 \\
\hline & $\begin{array}{l}\text { Correlación de } \\
\text { Pearson }\end{array}$ &, $911^{\star *}$ & 1 &, $755^{* *}$ &, $765^{* *}$ &, $797^{* *}$ &, $823^{* *}$ &,- 085 \\
\hline \multirow[t]{3}{*}{ C. Realización } & Sig. (bilateral) &, 000 & &, 000 &, 000 &, 000 &, 000 &, 549 \\
\hline & $\mathrm{N}$ & 55 & 55 & 55 & 55 & 55 & 55 & 52 \\
\hline & $\begin{array}{c}\text { Correlación de } \\
\text { Pearson }\end{array}$ &, $905^{\star *}$ &, $755^{\star *}$ & 1 &, $846^{\star *}$ &, $730^{\star *}$ &, $811^{* *}$ & , 180 \\
\hline \multirow[t]{3}{*}{ C. Involucramiento } & Sig. (bilateral) &, 000 &, 000 & &, 000 &, 000 &, 000 & ,202 \\
\hline & $\mathrm{N}$ & 55 & 55 & 55 & 55 & 55 & 55 & 52 \\
\hline & $\begin{array}{c}\text { Correlación de } \\
\text { Pearson }\end{array}$ &, $926^{\star *}$ &, $765^{\star *}$ &, $846^{* *}$ & 1 &, $825^{\star *}$ &, $785^{* *}$ &, 067 \\
\hline \multirow[t]{3}{*}{ C. Supervisión } & Sig. (bilateral) &, 000 &, 000 &, 000 & &, 000 &, 000 & ,639 \\
\hline & $\mathrm{N}$ & 55 & 55 & 55 & 55 & 55 & 55 & 52 \\
\hline & $\begin{array}{l}\text { Correlación de } \\
\text { Pearson }\end{array}$ &, $902^{* *}$ &, $797^{* *}$ &, $730^{\star *}$ &, $825^{\star *}$ & 1 &, $755^{\star *}$ &,- 221 \\
\hline \multirow[t]{3}{*}{ C. Comunicación } & Sig. (bilateral) &, 000 &, 000 &, 000 &, 000 & &, 000 &, 115 \\
\hline & $\mathrm{N}$ & 55 & 55 & 55 & 55 & 55 & 55 & 52 \\
\hline & $\begin{array}{c}\text { Correlación de } \\
\text { Pearson }\end{array}$ &, $914^{* *}$ &, $823^{* *}$ &, $811^{* *}$ &, $785^{\star *}$ &, $755^{\star *}$ & 1 & ,120 \\
\hline \multirow[t]{3}{*}{ C. Condiciones } & Sig. (bilateral) &, 000 &, 000 &, 000 &, 000 &, 000 & & ,396 \\
\hline & $\mathrm{N}$ & 55 & 55 & 55 & 55 & 55 & 55 & 52 \\
\hline & $\begin{array}{l}\text { Correlación de } \\
\text { Pearson }\end{array}$ &, 010 &,- 085 & , 180 &, 067 &,- 221 &, 120 & 1 \\
\hline \multirow[t]{2}{*}{ Desempeño } & Sig. (bilateral) & 944 &, 549 & ,202 & ,639 &, 115 & ,396 & \\
\hline & $\mathrm{N}$ & 52 & 52 & 52 & 52 & 52 & 52 & 52 \\
\hline
\end{tabular}

**. La correlación es significativa al nivel 0,01 (bilateral). 
En la Tabla 2, se muestra que sí se presenta relación significativa entre los 5 indicadores del clima laboral, no obstante, éstos no se relacionan significativamente con el desempeño laboral general. Respecto al desempeño laboral general el índice de correlación es negativo o inverso, es decir, que no existirá por ninguna razón posibilidad de pensar que un buen clima laboral general pueda condicionar un buen desempeño laboral general, ni viceversa, así como respecto a los indicadores del clima laboral. Lo cual está respaldado en la tendencia de los participantes del estudio, puesto que no quieren ser Jefes debido a beneficios o a influencias del ambiente que los obliguen a buscar un desempeño eficaz, es más, saben que su medio laboral presenta serias deficiencias en su sistema de compensaciones y no hay gratificaciones, por lo que ellos quieren justificar su desempeño por una motivación intrínseca, porque forma parte de sí mismos, el poder dar lo mejor de sí mismos, casi naturalmente.

\section{DISCUSIÓN}

El esclarecimiento de la no confirmación de las hipótesis de investigación específicas, es decir, a la no correlación de los indicadores del clima laboral con el desempeño laboral en general, tiene su principal plataforma de sustento en que el personal de salud perteneciente a una nación se compone de trabajadores que se localizan en etapas muy disímiles de sus vidas profesionales; laboran en distintos tipos de establecimientos de salud y lo hacen sujetos a condiciones y presiones en incesante evolución. Una de las causas por las que no se ha comprobado la hipótesis de investigación general es que los participantes desconocen o no han tenido experiencia o conocimiento a pesar de que se les ha explicado, no parecen haber comprendido, la importancia del proceso de la evaluación del desempeño, como nos explica Ivancevich (2005), sobre las fases de ésta, existen la identificación, la medición y la gestión, y es que en este sentido los trabajadores no han comprendido la importancia de la primera y tercera fase, y solo han cumplido con la medición, sin atender la precisión que debieron darle a estas evaluaciones. Además, según Bain (2003), señala que existen dos factores que pueden contribuir al mejoramiento de la productividad, y que son considerados dentro de los factores del desempeño laboral, estos son: factores internos y externos, de los cuales, los factores internos, poseen factores duros y blandos, precisamente el último aspecto es el que falta mejorar para posibilitar también la correlación entre clima laboral y desempeño laboral. Para Drovett (1992), la capacitación del trabajador, es un proceso de formación implementado por el área de recursos humanos, y posibilita mejoras constantes en el ambiente laboral, en este caso, el MINSA no tiene programas de formación continua para sus trabajadores, sino más bien, estos ocurren según la intención del Ministro de Salud o responsable. Este factor de capacitación es uno de los factores que influyen en el desempeño laboral, y que se ha dejado de lado.

Respecto a ver al clima organizacional como herramienta de gestión, Rodríguez en el 2001, afirma que el desarrollo de los individuos dentro de una organización puede verse afectado por el clima que perciben dentro de la misma, lo que quiere decir, que al no concretarse la hipótesis de investigación se nota de por sí que hay una falsa visión de lo que debería ser valorado por los trabajadores de salud en el clima laboral institucionalmente. Likert (1961), afirma que el clima organizacional tiene una influencia importante sobre las actitudes y comportamiento y el desempeño de los empleados dentro de las organizaciones (citado por Jaime y Araujo, 2007), y en base a ello, se ha planteado la presente investigación, pero también se advierte que una influencia importante no es generalizable a todos los contextos, como en este estudio, para los trabajadores de salud no es determinante por lo que se acepta la hipótesis de investigación nula.

Asimismo, tomando en cuenta lo mencionado por Litwin y Stringer (1968) lo cual significa que no se hallan bien estructuradas las organizaciones, aquellas propiedades dentro de la estructura del MINSA no son adecuadamente jerarquizadas o peor aún muy burocratizadas, por lo que también se puede explicar el rechazo a la hipótesis de investigación. Es también importante la influencia de ciertos factores, y sobre todo es necesario que se pueda trabajar de manera conjunta tanto el clima laboral como el desempeño laboral, y es que aunque puedan sonar discriminatorio inicialmente, se debe analizar minuciosamente todo proyecto en favor de esto, para tal caso se propone que se debería llevar un registro nacional de trabajadores de la salud, que sea visible a través de la ley de transparencia y acceso a la información pública, a fin de incentivar a que tales trabajadores laboren solo en una institución con un sueldo justo que no les obligue a buscar más trabajados y desconcentrarse de sus actividades de salud, en la gestión de un buen clima laboral, y por consiguiente una mejora en el desempeño laboral, entonces, en la medida de las posibilidades presupuestales se debería hacer un mejor pago por concepto de honorarios profesionales.

Asimismo, no tener planes y proyectos personales puede afectar en desmedro del trabajo, al verse agobiado solo por la rutina laboral, esto es un conflicto interno que les ocurre a varios trabajadores de la salud y no les permite cumplir completamente sus funciones. Esta relación clima desempeño, tampoco se da porque nuestro sistema de salud es precario, abandonado a su suerte o con toda crudeza "pobre", con un porcentaje mínimo del producto bruto interno. Para mejorar nuestro sistema se va hacer indispensable eliminar los puestos y cargos de confianza, en salud y a todo nivel. Una posibilidad de mejora, es también certificar y evaluar cada 5 años a los profesionales asistenciales, proveer de software específico por cada especialidad. Además, se le debe hacer sentir al trabajador que todos en el país tienen los mismos beneficios, por ejemplo, para no ir muy lejos en Juliaca, a los trabajadores del MINSA, se les proporciona una canasta navideña donde unos reciben sacos de arroz y de azúcar, entre muchas otros productos, comparados con los mismos puestos pero en Arequipa, que reciben muchísimo menos, es decir, hay que minimizar las brechas entre los propios trabajadores del MINSA. También, sabemos que hay ascensos en el sector salud y que estos son "movidos" muchas veces por influencias, eso debe desaparecer, y cada 4 años debe dársele la posibilidad de postular a un ascenso por medio de una evaluación a medida según sea cada puesto de trabajo funcional. Se exhorta igualmente a realizar estudios cualitativos en diferentes rubros de la salud y en sectores no antes vistos como por ejemplo en el sistema de salud para personas con capacidades diferentes (PCD). Sería importante determinar si factores como el contexto socio económico, 
la idiosincrasia y/o la cultura de la muestra explican la no confirmación de algunas hipótesis de investigación. Por lo que se sugiere estudios longitudinales y en otros entornos sociales, así como en empresas nacionales e internacionales.

En conclusión, se ha determinado que no existe relación significativa entre el clima laboral y el desempeño laboral en el personal de salud, involucrado en actividades administrativas de los Hospitales del MINSA de la Región Arequipa, sin embargo, se ha determinado que sí existe un alto grado de relación significativa entre los indicadores del clima laboral así como también entre los indicadores del desempeño laboral, es decir, que los instrumentos utilizados miden lo que realmente deben medir. Por consiguiente, se ha determinado que no existe relación entre los indicadores del clima laboral (autorrealización, involucramiento laboral, supervisión, comunicación y condiciones laborales) y el desempeño laboral en general.

Asimismo, para futuras investigaciones se dan las siguientes recomendaciones: en primer lugar: es necesario tomar en cuenta aspectos de clima laboral y desempeño laboral por separado, a través de documentos técnicos y guías prácticas en todas las organizaciones de salud; en segundo lugar: Es necesario mejorar el sistema de salud peruano en general, como en sus características particulares. Y finalmente, en tercer lugar: el valor que se le da a los profesionales de la salud es mínimo en comparación a los grandes sectores empresariales, por lo que esto debe cambiar notablemente.

\section{AGRADECIMIENTOS}

Agradezco a mi asesor el Dr. Bernardo de la Gala Velásquez, por sus conocimientos y sabiduría. Agradezco también al Dr. Gustavo Rondón Fudinaga, Gerente Regional de Salud del Gobierno Regional de Arequipa, quien brindo la autorización para la realización de mi tesis en los Hospitales de la Region Arequipa. Finalmente y en especial a la Universidad Nacional de San Agustin, CIENCIACTIVA y CONCYTEC por el financiamiento integro de la presente investigación.

\section{REFERENCIAS BIBLIOGRÁFICAS}

1. Bain, D. (1993). Productividad, la solución a los problemas de la empresa. México D.F.: McGraw-Hill.

2. Drovett, S. (1992). Dicersión de la Salud. Buenos Aires: Editorial Maechi.

3. Ivancevich, J. (2005). Administración de recursos humanos. México D.F.: Mc Graw-Hill.

4. Jaime, P. y Araujo, Y. (2003). Psicología de las Organizaciones: Problemas contemporáneos. México D.F.: Ed. Prentice Hall.

5. Likert, R. (1968). El factor humano de la empresa. Bilbao: Devito.

6. Litwin, G. H. y Stringer, R. A. (1968). Motivation and organizational climate. Boston: Harvard University.

7. Palma, S. (1999). Elaboración y Validación de Escala CL-SPC en trabajadores de Lima Metropolitana. Revista Teoría e Investigación en Psicología, 9(1), $27-$ 34.

8. Puchol, L. (2007). Dirección y Gestión de Recursos
Humanos. 7ma. Edición. Madrid: Ediciones Díaz de Santos.

9. Robbins, S. (1999). Comportamiento Organizacional. México D.F.: Prentice Hall.

10. Rodríguez, A. (2001). Diagnostico Organizacional. Mexico D.F.: Ediciones Alfaomega.

11. Smith, K. N., Gunzenhauser, J. D. y Fielding, J. E. (2010). Reinvigorating performance evaluation: first steps in a local health department. Public Health Nursing, 27(5), 425-432. 